\title{
Review of: "Revising the Role of Cortical Cytoskeleton During Secretion: Actin and Myosin XI Function in Vesicle Tethering"
}

\author{
$\mathrm{HAO} X U^{1}$ \\ 1 University of Southern Mississippi
}

Potential competing interests: The author(s) declared that no potential competing interests exist.

The authors of the manuscript investigated the functions of cytoskeletal proteins in vesicle tethering in plant cells by exploiting a highly sensitive and quantitative imaging method called single particle tracking. Using genetics and pharmacological inhibitors, the authors provide quite strong evidence that initial vesicle tethering step of exocytosis requires actin and myosin $\mathrm{XI}$, whereas cortical microtubules plays a minor role in the exocytic process. This reviewer finds the manuscript written in a clear and logical fashion, although it could use some improvements as described below.

1. While the phenotypes associated with actin and myosin XI mutants are exciting, it is not known whether these phenotypes can be rescued by re-introducing a wild-type allele. This would likely require generating a new line of Arabidopsis, which will make the conclusions more convincing.

2. To disrupt microtubule function, authors used Oryzalin, a herbicide that shows a differential effect on the subpellicular microtubules and microtubules of the mitotic spindle, disrupting both at $2.5 \mu \mathrm{M}$, but only the subpellicular microtubules at $0.5 \mu \mathrm{M}$ (Morrissette and Sibley, 2002). It is not clearly explained why Oryzalin was used at $20 \mu \mathrm{M}$ in this manuscript. The data would be strengthened should authors choose to test in parallel other chemicals that regulate microtubule dynamics (e.g., Colchicine, Taxol, etc).

3. In Fig 3B, microtubule filaments may have reduced in number upon oryazlin treatment but did not completely disappear. It is possible they are still required for vesicle delivery but their concentration is not rate limiting even upon oryzalin treatment.

4. It is not clearly how many biological repeats were conducted for Fig $1 \mathrm{E}$. 